\title{
UNIQUENESS OF HAAR SERIES WHICH ARE $(C, 1)$ SUMMABLE TO DENJOY INTEGRABLE FUNCTIONS
}

BY

WILLIAM R. WADE

ABSTRACT. A Haar series $\Sigma a_{k} x_{k}$ satisfies Condition $H$ if $a_{k} x_{k} / k \rightarrow 0$ uniformly as $k \rightarrow \infty$. We show that if such a series is $(C, 1)$ summable to a Denjoy integrable function $f$, except perhaps on a countable subset of $[0,1]$, then that series must be the Denjoy-Haar Fourier series of $f$.

1. Introduction. The Haar functions $\chi_{0}, \chi_{1}, \cdots$ are a complete orthonormal system in the Hilbert space $L^{2}[0,1]$. For the purposes of this paper we need only recall that $\chi_{0}$ is identically 1 and that, given any positive integer $n=2^{m}$ $+k$, where $0 \leq k<2^{m}$, the corresponding Haar function $\chi_{n}$ takes on the value $+\sqrt{2^{m}}$ on the open interval

$$
\Delta(1, n) \equiv\left(2 k / 2^{m+1},[2 k+1] / 2^{m+1}\right),
$$

and takes on the value $-\sqrt{2^{m}}$ on the open interval

$$
\Delta(2, n) \equiv\left([2 k+1] / 2^{m+1},[2 k+2] / 2^{m+1}\right) .
$$

Furthermore, the support of that $n$th Haar function is precisely the closure of the union of intervals (1) and (2):

$$
\operatorname{Supp}\left[\chi_{n}\right]=\left[k / 2^{m},[k+1] / 2^{m}\right]
$$

The Denjoy integral (see [5, pp. 84-85]), (D) $\int_{a}^{b}$, is more general than either Lebesgue's integral or the improper Riemann integral.

The D-Haar Fourier series of a Denjoy integrable function $f$ is a Haar series $S(x)=\sum_{k=0}^{\infty} a_{k} X_{k}(x)$ which is related to $f$ by the following formula:

$$
\alpha_{k}=(D) \int_{0}^{1} f(x) \chi_{k}(x) d x .
$$

If (3) holds and $f$ is also Lebesgue integrable, then $S$ is simply the Haar Four. ier series of $f$.

Presented to the Society, January 18, 1972; received by the editors February 22, 1972.

AMS (MOS) subject classifications (1969). Primary 4220, 4215, 4210; Secondary 4032, 4042.

Key words and phrases. Harr functions, Denjoy integral, $(C, 1)$ summable.

Copyright $\odot 1973$, American Mathematical Society 
The harmonic analysis of Haar series is greatly simplified by the following property of Haar Fourier series:

The Haar Fourier series of any Lebesgue integrable function $f$ converges to $f$ almost everywhere on $[0,1]$.

The standard proof of this fact [1, pp. 47-50] uses only one consequence of the Lebesgue integrability of $f$ : the derivative of an indefinite Lebesgue integral is equal to the integrand almost everywhere. Since this property is also shared by Denjoy's integral, that same proof will establish that The D-Haar Fourier series of $f$ converges to $f$ a.e.

2. The uniqueness theorem. In this section we state the uniqueness theorem proved in $\$ 4$ and relate it to the research presented in [2] and [4].

A Haar series $\sum_{k=0}^{\infty} a_{k} X_{k}(x)$ satisfies Condition $G$ if, given any $t_{0} \in[0,1]$,

$$
\lim _{j \rightarrow \infty} a_{K_{j}} / \chi_{K_{j}}\left(t_{0}\right)=0
$$

where $K_{1}, K_{2}, \cdots$ are all those indices $p$ for which $\chi_{p}\left(t_{0}\right) \neq 0$. Lemma 3 of [2] shows that a D-Haar Fourier series always satisfies Condition $G$.

A Haar series $\Sigma_{k=0}^{\infty} a_{k} \chi_{k}(x)$ satisfies Condition $H$ if

$$
\lim _{n \rightarrow \infty} a_{n} \chi_{n}(x) / n=0 \quad \text { uniformly for } x \in[0,1] \text {. }
$$

Condition $G$ is equivalent to supposing that the limit in (6) exists pointwise, since if $\chi_{p}\left(t_{0}\right) \neq 0$ then $p \geq\left|\chi_{p}^{2}\left(t_{0}\right)\right| \geq p / 8$. Thus Condition $H$ can be viewed as the uniform analogue of Condition $G$.

F. G. Arutjunjan [2] has shown that if a Haar series $S$ satisfying Condition $G$ converges, except perhaps on a countable subset of $[0,1]$, to a Denjoy integrable function $f$, then $S$ must be the D-Haar Fourier series of $f$.

We shall denote the $n t h$ partial $(C, 1)$ sum of a Haar series $S(x)=$ $\sum_{k=0}^{\infty} \alpha_{k} \chi_{k}(x)$ by

$$
\sigma_{n}(S ; x) \equiv \frac{S_{1}(x)+\cdots+S_{n+1}(x)}{n+1} \equiv \sum_{k=0}^{n}\left(1-\frac{k}{n+1}\right) \alpha_{k} x_{k}(x)
$$

where $S_{n+1}(x)=\sum_{k=0}^{n} a_{k} X_{k}(x)$. If the sequence displayed in (7) converges at $x$ then $S$ is said to be $(C, 1)$ summable at $x$. If $S$ converges at $x$ it is automatically $(C, 1)$ summable at $x$; the converse of this statement is false.

The question motivating this research was: Does Arutjunjan's result still hold if $S$ is only $(C, 1)$ summable off that countable set?

If $S$ also satisfies Condition $H$ (loosely, if $S$ satisfies Condition $G$ uniformly) the answer to this question is yes and is obtained as a corollary to the following result, which is proved in $\$ 4$. 
Theorem. Let

$$
S(x)=\sum_{k=0}^{\infty} \alpha_{k} \chi_{k}(x)
$$

be a Haar series satisfying Condition $H$ such that

$$
\limsup _{n \rightarrow \infty}\left|\sigma_{n}(S ; x)\right|<\infty
$$

for all but countably many $x^{\prime}$ 's in $[0,1]$. Suppose further that $f$ is a Denjoy inte. grable function sucb that

$$
\lim _{n \rightarrow \infty} \sigma_{n}(S ; x)=f(x) \text { a.e. in }[0,1] .
$$

Then $S$ is the D.Haar Fourier series of $f$.

This result is already known in the case that $f$ is Lebesgue integrable (see [4]), but the techniques used here to establish Lemma 4 in the next section are radically different and substantially more complex due to the fact that a function can be conditionally Denjoy integrable.

\section{Fundamental lemmas.}

Lemma 1. Let $S$ be a Haar series satisfying Condition $G$ and $\Delta\left(i_{0}, k_{0}\right)$ be an interval of the form (1) or (2) such that

$$
S_{k_{0}+1}(x) \not 0 \text { for } x \in \Delta\left(i_{0}, k_{0}\right) \text {. }
$$

Then given any $t_{0} \in[0,1]$ there is an interval $\Delta\left(i_{0}^{\prime}, k_{0}^{\prime}\right)$ of the form (1) or (2) such that $t_{0}$ does not lie in the closure of $\Delta\left(i_{0}^{\prime}, k_{0}^{\prime}\right)$ and such that

$$
S_{k_{0}^{\prime}+1}(x) \not 0 \text { for } x \in \Delta\left(i_{0}^{\prime}, k_{0}^{\prime}\right) \text {. }
$$

This lemma was proved on pp. 225-226 of [4].

Lemma 2. Let $f$ be a Denjoy integrable function and $\mathfrak{D}$ be any collection of nonoverlapping subintervals of a fixed interval $\Delta\left(i_{0}, k_{0}\right)$ of the form (1) or (2). Define the function $f^{*}$ by

$$
\begin{aligned}
f^{*}(x) & =f(x) \quad \text { if } \quad x \in \Delta\left(i_{0}, k_{0}\right) \ldots \cup \mathfrak{T}, \\
& =\frac{1}{|\Delta|}(D) \int_{\Delta} f(t) d t \quad \text { if } x \in \Delta \in \mathfrak{T} .
\end{aligned}
$$

Then the $\left(k_{0}+1\right)$ st partial sum of the D-Haar Fourier series $T$ of $f$ can be written as

$$
T_{k_{0}+1}(x)=\frac{1}{\left|\Delta\left(i_{0}, k_{0}\right)\right|}(D) \int_{\Delta\left(i_{0}, k_{0}\right)} f^{*}(t) d t,
$$

for any $x \in \Delta\left(i_{0}, k_{0}\right)$. 
This lemma was proved on pp. 338-339 of [2].

Lemma 3. Let $f$ be a Denjoy integrable function, $T$ be its D-Haar Fourier series and $\mathfrak{D}$ be any collection of nonoverlapping subintervals of a fixed interval $\Delta\left(i_{0}, k_{0}\right)$ of the form (1) or (2). Suppose further that $S^{*}$ is any Haar series whose partial $(C, 1)$ sums satisfy

$$
\lim _{n \rightarrow \infty} \int_{\Delta\left(i_{0}, k_{0}\right)} \sigma_{n}\left(S^{*} ; t\right) d t=(D) \int_{\Delta\left(i_{0}, k_{0}\right)} f^{*}(t) d t
$$

where $f^{*}$ is defined by (14). Then $S_{k_{0}+1}^{*}(x) \equiv T_{k_{0}+1}(x)$ for $x \in \Delta\left(i_{0}, k_{0}\right)$.

Proof. The integral of a Haar function is zero, and the support of each Haar function $\chi_{k_{0+1}}, \chi_{k_{0}+2}, \cdots$ is either a subset of $\Delta\left(i_{0}, k_{0}\right)$ or disjoint from it. Consequently, if $n>k_{0}$,

$$
\begin{aligned}
& \int_{\Delta\left(i_{0}, k_{0}\right)} \sigma_{n}\left(S^{*} ; t\right) d t \\
& \quad=\frac{k_{0}+1}{n+1} \int_{\Delta\left(i_{0}, k_{0}\right)} \sigma_{k_{0}}\left(S^{*} ; t\right) d t+\frac{n-k_{0}+1}{n+1} \int_{\Delta\left(i_{0}, k_{0}\right)} S_{k_{0}+1}^{*}(t) d t .
\end{aligned}
$$

But $\chi_{0}, \chi_{1}, \cdots, \chi_{k_{0}}$ are all constant on $\Lambda\left(i_{0}, k_{0}\right)$ so the last summand of (16) is simply

$$
\frac{n-k_{0}+1}{n+1}\left|\Delta\left(i_{0}, k_{0}\right)\right| S_{k_{0}+1}^{*}(x)
$$

for any $x \in \Lambda\left(i_{0}, k_{0}\right)$. Solving (16) for $S_{k_{0}+1}^{*}(x)$ we then obtain

$$
\begin{aligned}
S_{k_{0}+1}^{*}(x)= & \frac{n+1}{n-k_{0}+1} \cdot \frac{1}{\left|\Delta\left(i_{0}, k_{0}\right)\right|} \int_{\Delta\left(i_{0}, k_{0}\right)} \sigma_{n}\left(S^{*} ; t\right) d t \\
& -\frac{k_{0}+1}{n-k_{0}+1} \cdot \frac{1}{\left|\Delta\left(i_{0}, k_{0}\right)\right|} \int_{\Delta\left(i_{0}, k_{0}\right)} \sigma_{k_{0}}\left(s^{*} ; t\right) d t .
\end{aligned}
$$

Taking the limit of both sides of this equation as $n \rightarrow \infty$ and applying (15) results in

$$
S_{k_{0}+1}^{*}(x)=\frac{1}{\left|\Delta\left(i_{0}, k_{0}\right)\right|}(D) \int_{\Delta\left(i_{0}, k_{0}\right)} f^{*}(t) d t,
$$

for any $x \in \Lambda\left(i_{0}, k_{0}\right)$. According to Lemma 2 , this is the value of $T_{k_{0}+1}$ over $\Delta\left(i_{0}, k_{0}\right)$.

Lemma 4. Let $f$ be Denjoy integrable, $T$ be its Haar Fourier series and suppose that $S(x)=\Sigma a_{k} X_{k}(x)$ is a Haar series satisfying Condition H which is $(C, 1)$ summable to $f$ almost everywhere. Suppose further that on some fixed interval $\Delta\left(i_{0}, k_{0}\right)$ of the form (1) or (2) that

$$
S_{k_{0}+1}(x) \not \equiv T_{k_{D}+1}(x) \text { for } x \in \Delta\left(i_{0}, k_{0}\right) \text {. }
$$


Then given any $M>0$ there is an interval $\Delta\left(i_{k}^{\prime}, k_{0}^{\prime}\right)$ contained in $\Delta\left(i_{0}, k_{0}\right)$ such that $\left|\sigma_{k_{0}^{\prime}}(S ; x)\right|>M$ for $x \in \Delta\left(i_{0}^{\prime}, k_{0}^{\prime}\right)$ and such that $T_{k_{0+1}^{\prime}}$ and $S_{k_{0+1}^{\prime}}$ satisfy (17) on $\Delta\left(i_{0}^{\prime}, k_{0}^{\prime}\right)$.

Proof. We shall prove this lemma in six steps:

I. We begin by supposing the lemma is false; i.e., that there is an $M_{0}$ such that if $\Delta(j, n)$ is any subinterval of $\Delta\left(i_{0}, k_{0}\right)$ of the form (1) or (2) then

$$
\begin{aligned}
&\left|\sigma_{n}(S ; x)\right|>M_{0} \text { for } x \in \Delta(j, n) \\
& \text { implies } S_{n+1}(x) \equiv T_{n+1}(x) \text { for } x \in \Delta(j, n) .
\end{aligned}
$$

II. We next shall use Lemma 1 to show that it is no loss of generality to suppose that $\left|a_{n} \chi_{n}(x) / n\right|<M_{0}$ for $n \geq k_{0}$.

III. We shall then construct a "maximal" class of intervals

$$
\mathfrak{I} \equiv\left\{\Delta\left(i_{1}, \rho_{1}\right), \Delta\left(i_{2}, \rho_{2}\right), \ldots\right\}
$$

on which $S_{\rho_{k+1}}$ and $T_{\rho_{k+1}}$ are identically equal.

IV. Next we shall construct a subseries $S^{*}$ of $S$ such that.
(a) $S^{*}$ is $(C, 1)$ summable to the function $f^{*}$ almost everywhere,

where $f^{*}$ is defined with respect to the class $\mathscr{D}$ chosen in III by (14);

(b) $S^{*}$ and $S$ are identical in $\Delta\left(i_{0}, k_{0}\right) \sim \mathcal{D}$;

- (c) $S_{k_{0}+1}^{*}(x) \equiv S_{k_{0}+1}(x)$ for $x \in \Delta\left(i_{0}, k_{0}\right)$.

V. We shall then show that the partial $(C, 1)$ sums of this series $S^{*}$ are bounded by $13 M_{0}$ by showing that if they are not, we are lead to a contradiction of the construction of $S^{*}$ in step IV.

VI. Finally, we shall use IV and V to lead to the ultimate contradiction. Indeed, since any $(C, 1)$ partial sum of a Haar series is Lebesgue integrable, we can use IV(a) and V to conclude that $f^{*}$ is Lebes gue integrable and that (15) holds. Consequently, by Lemma $3, s_{k_{0}+1}^{*} \equiv T_{k_{0}+1}$ on $\Delta\left(i_{0}, k_{0}\right)$. But, by IV(c), this identity also implies $S_{k_{0+1}} \equiv T_{k_{0}+1}$ on $\Delta\left(i_{0}, k_{0}\right)$. Since this and hypothesis (17) of this lemma are incompatible, assumption I was false; i.e., the proof of the lemma is complete by contradiction.

What remains, then, is to execute steps II through V:

II. By (6) we choose an integer $2^{Q}$ so large that

$$
\left|\alpha_{n} \chi_{n}(x) / n\right|<M_{0} \quad \text { whenever } n \geq 2^{Q}
$$

and for any $x \in[0,1]$.

Using Lemma 1 successively on the points $t_{0}=1 / 2^{Q}, t_{0}=2 / 2^{Q}, \cdots, t_{0}$ $=\left(2^{Q}-1\right) / 2 Q$ and the series $S-T$, we may suppose with no loss of generality that $\left|\Delta\left(i_{0}, k_{0}\right)\right|<1 / 2^{Q}$. This fact together with (19) will assure us that

$$
\left|a_{n} \chi_{n}(x) / n\right|<M_{0} \text { for } n \geq k_{0}
$$

and for any $x \in[0,1]$. 
III. If there is no subinterval $\Delta\left(i_{1}, \rho_{1}\right)$ of $\Delta\left(i_{0}, k_{0}\right)$ such that $S_{\rho_{1+1}} \equiv$ $T_{\rho_{1+1}}$ on $\Delta\left(i_{1}, \rho_{1}\right)$ then set $\mathscr{D}=\varnothing$. Otherwise let $\Delta\left(i_{1}, \rho_{1}\right)$ be the first such interval of the form (1) or (2).

Suppose that we have either terminated this process or have managed to choose $N-1$ intervals $\Delta\left(i_{1}, \rho_{1}\right), \ldots, \lambda\left(i_{N-1}, \rho_{N-1}\right)$. If there is no subinterval $\Delta\left(i_{n}, \rho_{n}\right)$ of $\Delta\left(i_{0}, k_{0}\right)$ disjoint from $\bigcup_{l=1}^{N-1} \Delta\left(i_{l}, \rho_{l}\right)$ such that $S_{\rho_{n}+1} \equiv T_{\rho_{n}+1}$ then set

$$
\mathfrak{L}=\left\{\Delta\left(i_{l}, \rho_{l}\right): l=1,2, \cdots, N-1\right\} .
$$

Otherwise let $\Delta\left(i_{N}, \rho_{N}\right)$ be the first such interval of the form (1) or (2).

If this process can be continued indefinitely, set

$$
\mathfrak{I}=\left\{\Delta\left(i_{l}, \rho_{l}\right): l=1,2, \cdots\right\} .
$$

Clearly $\mathfrak{D}$ is a collection of nonoverlapping intervals of the form (1) or (2). Define the function $f^{*}$ by (14). Notice that if $\mathcal{D}$ is empty then $f^{*}$ is identically equal to $f$.

IV. We s hall construct the series $S^{*}$ by choosing a particular sequence of integers $n_{1}<n_{2}<\cdots$ which are indices of Haar functions whose support lies in the closure of $\Delta\left(i_{0}, k_{0}\right)$.

Let $n_{1}$ be that integer such that $\Delta\left(1, n_{1}\right) \cup \Delta\left(2, n_{1}\right)$ is the interval $\Delta\left(i_{0}, k_{0}\right)$ without its midpoint. For instance, if $\Delta\left(i_{0}, k_{0}\right)=(1 / 4,1 / 2)$ then $n_{1}=5$. By (17), $n_{1}$ is the first integer such that $S_{k_{0+1}} \not \equiv T_{k_{0+1}}$ on $\Delta\left(i_{0}, k_{0}\right)$.

Let $n_{2}$ be the very next integer such that the support of $\chi_{n_{2}}$ lies in the closure of $\Delta\left(i_{0}, k_{0}\right)$ and such that, if $i_{1}$ and $k_{1}$ are chosen so that $\Delta\left(1, n_{2}\right) \cup \Delta\left(2, n_{2}\right)$ is the interval $\Delta\left(i_{1}, k_{1}\right)$ without its midpoint, then $S_{k_{1}+1} \not \equiv T_{k_{1}+1}$ on $\Delta\left(i_{1}, k_{1}\right)$. Throughout the following pages we shall denote the interval $\Delta(j, n)$ without its midpoint as $\Delta^{*}(j, n)$.

We continue this process as long as possible, thereby generating subintervals $\Delta\left(i_{j}, k_{j}\right)$ of $\Delta\left(i_{0}, k_{0}\right)$ and integers $n_{j}(j=1,2, \ldots)$ such that

and

$$
S_{k_{j}+1} \not \equiv T_{k_{j}+1} \text { on } \Delta\left(i_{j}, k_{j}\right) \text { for } j=0,1,2, \ldots \text {. }
$$

Finally, using the sequence $n_{1}, n_{2}, \cdots$ just generated we set

$$
S^{*}(x) \equiv S_{k_{0}+1}(x)+\sum_{j=1}^{\infty} a_{n_{j}} \chi_{n_{j}}(x) .
$$

In case the process for selecting the $n_{j}$ 's terminates after a finite number of steps, $S^{*}$ is just a finite series. Note also that $\operatorname{IV}(\mathrm{c})$ is trivially satisfied. 
We shall now show IV(a) and (b) are also satisfied by this $S^{*}$.

Indeed, by the disjointness of the collection $D$ and by the choice of the sequence $\left\{n_{j}\right\}$, if the support of $\chi_{n}$ is contained in $\Delta\left(i_{0}, k_{0}\right)$ but $\chi_{n}$ does not appear in the sum (23), then it must be the case that Supp $\left(\chi_{n}\right) \subseteq \Delta\left(i_{l}, \rho_{l}\right)$ for some $l$. In particular, the series $S$ and $S^{*}$ are the same series in the set $\Delta\left(i_{0}, k_{0}\right) \sim \bigcup \mathcal{I}$. By the hypotheses of this lemma, then

(24) $\lim _{n \rightarrow \infty} \sigma_{n}\left(s^{*} ; x\right) \equiv f(x)=f^{*}(x)$ for almost every $x$ in $\Delta\left(i_{0}, k_{0}\right) \sim \bigcup \mathfrak{D}$.

On the other hand, if $x_{0} \in \Delta\left(i_{l}, \rho_{l}\right)$ for some $l$ used to define $\mathscr{L}$, then the disjointness of $\mathscr{D}$ means that the series (23) must be truncated at $\rho_{l}$. Since $S$ and $S^{*}$ were identical up to that point, $S^{*}\left(x_{0}\right) \equiv S_{\rho_{l+1}}\left(x_{0}\right)$. But by the choice of $\rho_{l}, S_{\rho_{l+1}} \equiv T_{\rho_{l+1}}$ on $\Delta\left(i_{0}, \rho_{0}\right)$. Lemma 2 and the fact $f^{*}$ is constant on $\Delta\left(i_{l}, \rho_{l}\right)$ now imply that $T \rho_{\rho_{l}+1} \equiv f^{*}$ on $\Delta\left(i_{l}, \rho_{l}\right)$. Combining these three facts we conclude that $S^{*}\left(x_{0}\right)=f^{*}\left(x_{0}\right)$. Since $x_{0}$ was any point in any interval of $\mathscr{D}$, we can now conclude that

$$
\left.\lim _{n \rightarrow \infty} \sigma_{n}\left(S^{*} ; x\right)=f^{*}(x) \text { on each } \Delta \in \mathscr{X}\right)
$$

Combining (24) and (25) we have IV(a).

V. Suppose that the partial $(C, 1)$ sums of $S^{*}$ are not bounded by $13 M_{0}$ on $\Delta\left(i_{0}, k_{0}\right)$ and let $L$ be the smallest index greater than or equal to $n_{1}$ such that $\left|\sigma_{L}\left(S^{*} ; t_{0}\right)\right|>13 M_{0}$ for some $t_{0} \in \Delta\left(i_{0}, k_{0}\right)$.

If we let $n_{p}$ be the largest number in the sequence $n_{1}, n_{2}, \ldots$ which is less than or equal to $L$, then for some choice of $j_{p}=1$ or 2 ,

$$
\left|\sigma_{L}\left(S^{*} ; x\right)\right|>13 M_{0} \text { for } x \in \Delta\left(j_{p}, n_{p}\right) .
$$

Indeed, if $L=n_{1}$ then (26) is trivial by (21). Otherwise we use the least property of $L$.

We first begin by noting that

$$
S_{n_{p}+1} \not T_{n_{p}+1} \text { on } \Delta^{*}\left(i_{p-1}, k_{p-1}\right) \text {. }
$$

Indeed, if (27) were false, then $S_{n_{p}+1}=S_{k_{p-1}+1}+a_{n_{p}} X_{n_{p}}$, and a corresponding equation involving $T$ and its $n_{p}$ th coefficient, say $\beta_{n_{p}}$, implies $S_{k_{p-1}+1}-$ $T_{k_{p-1+1}} \equiv\left(\beta_{n_{p}}-a_{n_{p}}\right) \chi_{n_{p}}$ on $\Delta^{*}\left(i_{p-1}, k_{p-1}\right)$. But $\chi_{n_{p}}$ changes signs in that punctured interval while the left-hand side of the above identity is constant in that punctured interval. The only possibility, then, is that $\beta_{n_{p}}=a_{n_{p}}$ which in turn forces $S_{k_{p-1+1}}-T_{k_{p-1+1}} \equiv 0$ on $\Delta^{*}\left(i_{p-1}, k_{p-1}\right)$. Since both partial sums are constant throughout $\Delta\left(i_{p-1}, k_{p-1}\right)$, this statement and (22) are incompatible; consequently (27) does hold. 
Let $j_{p}^{\prime} \neq j_{p}$ with $j_{p}^{\prime}=1$ or 2 . Then, by $(21), \Delta\left(j_{p}, n_{p}\right) \cup \Delta\left(j_{p}^{\prime}, n_{p}\right)=$ $\Delta^{*}\left(i_{p-1}, k_{p-1}\right)$; and, by (22), $s_{k_{p-1+1}} \not T_{k_{p-1}+1}$ on $\Delta\left(i_{p-1}, k_{p-1}\right)$. Hence by the contrapositive of (18),

$$
\left|\sigma_{k_{p-1}}(S ; x)\right| \leq M_{0} \text { for } x \in \Delta\left(i_{p-1}, k_{p-1}\right) .
$$

Note also by $(22)$ and the fact that $S$ and $S^{*}$ are identical outside $\mathscr{D}$, we have

$$
\sigma_{n}(S ; x) \equiv \sigma_{n}\left(S^{*} ; x\right) \quad \text { for } x \in \Delta\left(i_{p-1}, k_{p-1}\right)
$$

whenever $n=k_{p-1}, k_{p-1}+1, \cdots, n_{p+1}-1$. Consequently, the above inequality becomes

$$
\left|\sigma_{k_{p-1}}\left(S^{*} ; x\right)\right| \leq M_{0} \text { for } x \in \Delta\left(i_{p-1}, k_{p-1}\right) .
$$

Using (27) and the contrapositive of (18) we can also conclude that on at least one of the intervals $\Delta\left(j_{p}, n_{p}\right), \Delta\left(j_{p}^{\prime}, n_{p}\right)$,

$$
\left|\sigma_{n_{p}}\left(S^{*} ; x\right)\right| \leq M_{0} .
$$

But

$$
\begin{aligned}
\sigma_{n_{p}}\left(S^{*} ; x\right) & =\sum_{k=0}^{n_{p}-1}\left(1-\frac{k}{n_{p}+1}\right) \alpha_{k} \chi_{k}(x)+\frac{\alpha_{n_{p}} \chi_{n_{p}}(x)}{n_{p+1}} \\
& \equiv \Sigma_{1}(x)+\frac{\alpha_{n_{p}} \chi_{n_{p}}(x)}{n_{p+1}} .
\end{aligned}
$$

Hence by (20) and the triangle inequality,

$$
\left|\Sigma_{1}(x)\right| \leq\left|\sigma_{n_{p}}\left(S^{*} ; x\right)\right|+\left|\alpha_{n_{p}} \chi_{n_{p}}(x) /\left(n_{p}+1\right)\right| \leq M_{0}+M_{0}=2 M_{0}
$$

on at least one of the intervals $\Lambda\left(j_{p}, n_{p}\right), \Lambda\left(j_{p}^{\prime}, n_{p}\right)$. But $\Sigma_{1}$ is constant throughout the union of both these intervals, so $\left|\Sigma_{1}(x)\right| \leq 2 M_{0}$ for $x \in \Delta\left(i_{p-1}, k_{p-1}\right)$. Applying (20) and the triangle inequality again, we conclude that

$$
\left|\sigma_{n_{p}}\left(S^{*} ; x\right)\right| \leq 3 M_{0} \quad \text { for } x \in \Delta\left(i_{p-1}, k_{p-1}\right) .
$$

We shall now complete the proof of step V by showing that (28), (29) and (26) are incompatible with (27).

The definition of $(C, 1)$ sums and the construction of $S^{*}$ allow us to write the following equations for any $x \in \Delta\left(i_{p-1}, k_{p-1}\right)$.

$$
\sigma_{L}\left(S^{*} ; x\right)=\frac{n_{p}+1}{L+1} \sigma_{n_{p}}\left(S^{*} ; x\right)+\frac{L-n_{p}+1}{L+1} S_{n_{p}+1}^{*}(x)
$$




$$
\sigma_{L}\left(S^{*} ; x\right)=\frac{k_{p-1}+1}{L+1} \sigma_{k_{p-1}}\left(S^{*} ; x\right)+\frac{L-k_{p-1}+1}{L+1} S_{n_{p}}^{*}(x)
$$

$$
\begin{gathered}
+\frac{L-n_{p}+1}{L+1} \alpha_{n_{p}} \chi_{n_{p}}(x) ; \\
\sigma_{n_{p}}\left(S^{*} ; x\right)=\frac{k_{p-1}+1}{n_{p+1}} \sigma_{k_{p-1}}\left(S^{*} ; x\right)+\frac{n_{p}-k_{p-1}+1}{n_{p+1}} S_{n_{p}}^{*}(x)+\frac{\alpha_{n_{p}} \chi_{n_{p}}(x)}{n_{p+1}} .
\end{gathered}
$$

Since $\chi_{n_{p}}$ is constant on $\Delta\left(j_{p}, n_{p}\right)$ there are two possible cases:

or

$$
\frac{L-n_{p}+1}{L+1}\left|\alpha_{n_{p}} \chi_{n_{p}}(x)\right|>M_{0} \text { for } x \in \Delta\left(j_{p}, n_{p}\right)
$$

$$
\frac{L-n_{p}+1}{L+1}\left|\alpha_{n_{p}} \chi_{n_{p}}(x)\right| \leq M_{0} \text { for } x \in \Delta\left(j_{p}, n_{p}\right) \text {. }
$$

If (34) holds then we use (29) and (26) on (30) to conclude that

$$
\frac{L-n_{p}+1}{L+1}\left|S_{n_{p}+1}^{*}(x)\right|>10 M_{0} \text { on } \Delta\left(j_{p}, n_{p}\right) \text {. }
$$

But $S_{n_{p+1}}^{*}=S_{n_{p}}^{*}+\alpha_{n_{p}} \chi_{n_{p}}$, so (34) implies

$$
\frac{L-n_{p}+1}{L+1}\left|S_{n_{p}}^{*}\right|>10 M_{0}-M_{0}=9 M_{0} \text { on } \Delta\left(j_{p}, n_{p}\right) \text {. }
$$

Since $S_{n_{p}}^{*}$ is constant on $\Delta\left(i_{p-1}, k_{p-1}\right)$ this inequality must hold throughout the larger interval:

$$
\frac{L-n_{p}+1}{L+1}\left|S_{n_{p}}^{*}(x)\right|>9 M_{0} \text { for } x \in \Delta\left(i_{p-1}, k_{p-1}\right) .
$$

On the other hand, if (35) holds, then using (28) on (31) implies

$$
\left|\frac{L-k_{p-1}+1}{L+1} S_{n_{p}}^{*}(x)+\frac{L-n_{p}+1}{L+1} a_{n_{p}} \chi_{n_{p}}(x)\right|>12 M_{0} .
$$

on $\Lambda\left(i_{p \ldots 1}, k_{p \ldots 1}\right)$, which by (35) guarantees

$$
\frac{L-k_{p-1}+1}{L+1}\left|S_{n_{p}}^{*}\right|>11 M_{0}>9 M_{0} \text { on } \Delta\left(j_{p}, n_{p}\right) .
$$

This show that (36) holds in any case.

Now, $2 n_{p}$ is an element of the sequence $n_{1}, n_{2}, \cdots$ by (27). Hence by the choice of $n_{p}$ relative to $L, L<2 n_{p}$. The construction of the sequence $k_{j}$ shows that $2 k_{p-1} \leq n_{p}$. These two in equalities together yield $3\left(n_{p}-k_{p-1}+1\right)$ $>L-k_{p-1}+1$. Consequently, applying (20), (28), and (36) to (32), we conclude $\left|\sigma_{n_{p}}\left(S^{*} ; x\right)\right|>(1 / 3) 9 M_{0}-M_{0}-M_{0}=M_{0}$ for $x \in \Delta\left(i_{p-1}, k_{p-1}\right)$. This, together with (18) and (21), implies $S_{n_{p}+1} \equiv T_{n_{p}+1}$ on $\Delta^{*}\left(i_{p-1}, k_{p-1}\right)$. By (27) this is impossible. 
This final contradiction completes the proof of step $\mathrm{V}$ which in turn completes the proof of this lemma.

4. The proof of the theorem. Let $\left\{Z_{1}, Z_{2}, \cdots\right\}$ be the set of points in $[0,1]$ where

$$
\limsup _{n \rightarrow \infty}\left|\sigma_{n}(S ; x)\right|=+\infty .
$$

Suppose that $T$ is the D-Haar Fourier series of $f$, but that the theorem is false. Choose $k_{0}$ least so that the $k_{0}$ th Fourier coefficient of $f$ is different from $\alpha_{k_{0}}$. Clearly, then, $S_{k_{0+1}} \not T_{k_{0+1}}$. Since $T$ satisfies Condition $G$ (Lemma 3 of [2]) the series $S-T$ satisfies the hypotheses of Lemma 1. The series $S$ and $T$ also satisfy the hypotheses of Lemma 4.

Applying Lemmas 1 and 4 countably many times, we can thus choose a sequence of intervals $\Delta\left(i_{1}, k_{1}\right), \cdots, \Delta\left(i_{N}, k_{N}\right), \cdots$ of the form (1) or (2) such that

and

$$
Z_{n} \text { does not lie in the closure of } \Delta\left(i_{N}, k_{N}\right) \text {, }
$$

$$
\text { the closure of } \Delta\left(i_{N}, k_{N}\right) \text { is a subset of } \Delta\left(i_{N+1}, k_{N+1}\right) \text {, }
$$

$$
\left|\sigma_{k_{N}}(S ; x)\right|>N \quad \text { for } x \in \Delta\left(i_{N}, k_{N}\right) \text {, for } N=1,2, \ldots .
$$

By (38), $\bigcap_{N=1}^{\infty} \Delta\left(i_{N}, k_{N}\right)$ is not empty; let $\xi$ be in this intersection.

By (37), $\xi \notin\left\{Z_{1}, Z_{2}, \cdots\right\}$ which, by the definition of this sequence, implies

$$
\limsup _{n \rightarrow \infty}\left|\sigma_{n}(S ; \xi)\right|<\infty \text {. }
$$

Yet by (39), since $\xi \in \Delta\left(i_{N}, k_{N}\right)$ for all $N$, lim $\sup _{n \rightarrow \infty}\left|\sigma_{n}(s ; \xi)\right|=\infty$. This being incompatible with (40) completes the proof of the theorem by contradiction.

\section{BIBLIOGRAPHY}

1. G. Alexits, Konvergenzprobleme der Orthogonalreihen, Akad. Kiadó, Budapest, 1960; English transl., Internat. Series of Monographs in Pure and Appl. Math., vol. 20, Pergamon Press, New York, 1961. MR 28 \#5292; MR 36 \#1911.

2. F. G. Arutjunjan, Reconstruction of the coefficients of series with respect to Haar and Walsh systems convergent to functions integrable in the sense of Denjoy, Izv. Akad. Nauk SSSR Ser. Mat. 30 (1966), 325-344. (Russian) MR 33 \#1650.

3. W. R. Wade, A uniqueness theorem for Haar and Walsh series, Trans. Amer. Math. Soc. 141 (1969), 187-194. MR 39 \#4587.

4. - Uniqueness theory for Cesaro summable Haar series, Duke Math. J. 38 (1971), 221-227.

5. A Zygmund, Trigonometrical series. Vol. II, Cambridge Univ. Press, New York, 1959. MR $21 \# 6498$.

DEPARTMENT OF MATHEMATICS, UNIVERSITY OF TENNESSEE, KNOXVILLE, TENNES- 